\title{
Streptoccocus faecalis endocarditis: an emerging problem?
}

\author{
Philip D. Welsby \\ M.B., B.S., M.R.C.P., M.R.C.S., L.R.C.P. \\ Department of Infectious Diseases, The Royal Free Hospital, London, and \\ Foresterhill and Associated Hospitals, Aberdeen
}

\section{Summary}

The clinical features of 16 patients with Streptococcus faecalis endocarditis are reviewed and the currently favoured antimicrobial regimens for such infections are detailed.

\section{Introduction}

A recent 16-year retrospective survey of infective endocarditis in an area of North-East Scotland revealed the causative organism to be Streptococcus faecalis in sixteen out of ninety-one episodes (Welsby, 1977). In view of this high proportion it was thought worth-while to report some further interesting observations.

\section{Method}

The case notes of all patients recorded as having infective endocarditis between the years 1959 and 1974 inclusive were studied, and the notes of those with $S$. faecalis endocarditis were reviewed in particular detail. In each case the clinical diagnosis was confirmed by two or more positive blood cultures.

\section{Results}

Sixteen patients had $S$. faecalis endocarditis, with post-mortem confirmation being obtained in all nine who died.

The mean age was 59 years with a range of $21-80$ years and a median of 62 years (Fig. 1). Eleven of the sixteen patients were male.

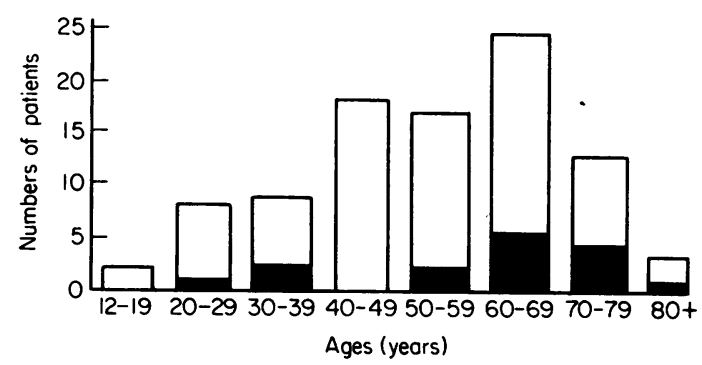

Fig. 1. The age groups of patients with infective endocarditis. All patients (in white) and patients with Streptococcus faecalis (in black).
All sixteen patients had previous hospital notes, but only three had previously known heart pathology before clinical examination at the time of admission.

\section{Predisposing factors}

One patient with diabetes had a $S$. faecalis urinary tract infection on admission. One patient had a trans-uretheral resection of the prostate and another had given birth before developing endocarditis. One further patient had a colonic carcinoma. Only one patient had received dental treatment within the 6 months before diagnosis.

\section{Heart valve involvement}

The aortic valve was involved clinically in nine patients (eight of whom were male and six of whom had no previously known heart pathology), the mitral valve alone in five patients and both valves were involved in two patients. Only five patients had a change in heart murmur recorded: all were male and all had developing aortic incompetence.

\section{Discussion}

$S$. faecalis thus frequently affected elderly patients (especially males with no history of heart pathology) and patients with isolated aortic valve involvement.

It seems likely that the patients under study had previously undetectable heart valve abnormality, especially as only three patients had known heart pathology. The ability of enterococci to attack normal heart valves is well documented (Geraci and Martin, 1954; Toh and Ball, 1960). It therefore seems sensible that, after a suspicion or clinical diagnosis of bacterial endocarditis in such patients, a faecal streptococcus should be considered a possible, indeed probable causative organism. Even if blood cultures were then sterile and non-bacterial causes of endocarditis excluded the 'best guess' treatment should include an antibacterial agent or combination of agents effective against $S$. faecalis. The currently favoured regimens are a combination of an aminoglycoside and penicillin or a penicillin derivative. Successful combinations include penicillin plus gentamicin (Weinstein and Moellering, 1973), 
but recent work suggests that ampicillin or amoxycillin in combination with an aminoglycoside is preferable if the aminoglycoside sensitivity of the organism is known (Basker and Sutherland, 1977). Combination therapy with amoxycillin seems particularly attractive as this drug produces serum levels almost twice those obtained with ampicillin and seems to be absorbed equally well in the fasting and non-fasting state (Sutherland, Croydon and Rolinson, 1972). Amoxycillin has to be given orally and, as with most drugs given orally, there may be unpredictability of absorption but in practice oral therapy of endocarditis has been successful (Gray, 1975). The choice of aminoglycoside appears to rest between gentamicin, tobramycin and amikacin as Basker and Sutherland (1977) reported about half their strains of enterococci (which had been isolated mainly from patients with endocarditis) to be highly resistant to streptomycin and one third of these isolates were also resistant to kanamycin.

Whichever drugs are used in the therapy of $S$. faecalis endocarditis, their mean inhibitory and bactericidal concentrations necessary to treat a particular patient's organism should be established, the blood levels of the drug concerned should also be monitored to ensure that adequate levels are actually being obtained and the actual activity of the drug against the specific organism confirmed by means of a 'back-titration' of the organism against dilutions of the patient's serum whilst receiving therapy.

It should be emphasized that one should not hesitate to use the potentially toxic aminoglycosides in older patients as here reported, provided serum levels are assayed regularly and renal function monitored (as this is the major route of aminoglycoside excretion), especially as the urinary tract is at particular risk of being compromised providing as it does the most common source of the faecal streptococcal organisms causing endocarditis (Geraci and Martin, 1954; Toh and Ball, 1960).

\section{Acknowledgment}

I thank the Physicians' Committee of the Aberdeen Hospitals for their permission to inspect the case notes of patients admitted under their care.

\section{References}

BASKer, M.J. \& Sutherland, R. (1977) Activity of amoxycillin, alone, and in combination with aminoglycoside antibiotics against streptococci associated with bacterial endocarditis. Journal of Antimicrobial Chemotherapy, 3, 273.

GeraCI, J.E. \& MARTIN, W.J. (1954) Antibiotic therapy of bacterial endocarditis: subacute enterococcal endocarditis; clinical, pathologic and therapeutic consideration of 33 cases. Circulation, 10, 173.

GRAY, I.R. (1975) The choice of antibiotic for treating infective endocarditis. Quarterly Journal of Medicine, 175, 449.

Sutherland, R., Croydon, E.A.P. \& Rolinson, G.N. (1972) Amoxycillin: a new semi-synthetic penicillin. British Medical Journal, 2, 13.

ToH, C.C.S. \& BALL, K.P. (1960) Natural history of Streptococcus faecalis endocarditis. British Medical Journal, 2, 640.

Weinstein, A.J. \& Moellering JR, R.C. (1973) Penicillin and gentamicin therapy for enterococcal infections. Journal of the American Medical Association, 223, 1030.

WelsBY, P.D. (1977) Infective endocarditis - a retrospective study. Practitioner, 218, 382. 